\title{
Long-Term Safety and Effectiveness of Adalimumab for Moderate to Severe Psoriasis: Results from 7-Year Interim Analysis of the ESPRIT Registry
}

\author{
Alan Menter (D) Diamant Thaçi · Jashin J. Wu • William Abramovits • \\ Francisco Kerdel · Dilek Arikan · Dianlin Guo • Arijit Ganguli • \\ Mareike Bereswill · Anne Camez · Wendell C. Valdecantos
}

Received: June 20, 2017 / Published online: August 16, 2017

(C) The Author(s) 2017. This article is an open access publication

\section{ABSTRACT}

Introduction: ESPRIT (NCT00799877) is an ongoing 10-year international prospective observational registry evaluating the long-term safety and effectiveness of originator adalimumab in routine clinical practice for adult

Enhanced content To view enhanced content for this article, go to www.medengine.com/Redeem/ 757AF0604D62602C.

\section{A. Menter $(\square)$}

Division of Dermatology, Baylor University Medical Center, Dallas, TX, USA

e-mail: amderm@gmail.com

D. Thaçi

Comprehensive Center for Inflammation Medicine, University Medical School Schleswig-Holstein,

Campus Lübeck, Germany

J. J. Wu

Kaiser Permanente Los Angeles Medical Center, Los Angeles, CA, USA

W. Abramovits

Dermatology Treatment and Research Center,

Dallas, TX, USA

F. Kerdel

Florida Academic Dermatology Centers, Miami, FL, USA

D. Arikan - D. Guo - A. Ganguli · W. C. Valdecantos AbbVie Inc, North Chicago, IL, USA

M. Bereswill · A. Camez

AbbVie Deutschland GmbH \& Co KG,

Ludwigshafen, Germany patients with chronic plaque psoriasis. Herein, we report the long-term safety, effectiveness, and patient-reported outcomes (PROs) following adalimumab treatment over the first 7 years of the ESPRIT registry.

Methods: All treatment-emergent (All-TE) adverse events (AE) since the initial (first ever) dose of adalimumab were assessed. Physician Global Assessment (PGA) and PROs (PROs for US patients only) were evaluated during registry participation.

Results: As of 30 November 2015, 6051 patients in the ESPRIT registry were analyzed, representing 23,660.1 patient-years (PY) of overall adalimumab exposure. The incidence rates for All-TE serious AEs, serious infections, and malignancies were $4.4,1.0$, and 1.0 events per $100 \mathrm{PY}$ (E/100PY), respectively. The standardized mortality ratio for TE deaths in the registry was 0.27 (95\% CI 0.18-0.38). During the registry's first 7 years, PGA "clear" or "minimal" was achieved by $>50 \%$ of patients at each annual visit, and among US patients, the mean improvement from baseline in different PROs was maintained.

Conclusion: No new safety signals were identified during the first 7 years of the registry, and safety was consistent with the known safety profile of adalimumab. The number of $\mathrm{TE}$ deaths was below the expected rate. During the registry's first 7 years, most of the patients remained free of All-TE cardiovascular events, serious infections, and malignancy. As-observed 
effectiveness of adalimumab and improvements from baseline in PROs were maintained through 7 years of registry participation.

Funding: Abbvie.

Trial Registration: ClinicalTrials.gov identifier, NCT00799877.

Keywords: Adalimumab; Cardiovascular events; Effectiveness; Long-term safety; Malignancy; Patient-reported outcomes; Psoriasis; Registry; Serious infections

\section{INTRODUCTION}

Psoriasis, a chronic, immune-mediated, inflammatory skin disease, is prevalent in about $2-3 \%$ of the world's population $[1,2]$. Patients with psoriasis are at a higher risk of developing several comorbidities such as cardiovascular disease, metabolic syndrome, joint disease, depression, and Crohn's disease, resulting in diminished quality of life [3-7]. In addition, patients with psoriasis are at an increased risk for serious infections and certain malignancies compared to the general population [8-10]. Although topical therapies, phototherapy, or conventional systemic non-biologic therapies are most often used as the first line of treatment, the introduction of biologic agents has vastly improved disease management in patients with moderate-to-severe psoriasis [2, 11].

Adalimumab, a fully human recombinant, monoclonal antibody directed against tumor necrosis factor-alpha (TNF- $\alpha$ ), is approved for the treatment of moderate-to-severe chronic plaque psoriasis in adult patients, who are candidates for systemic therapy or phototherapy [12]. Long-term safety and efficacy of adalimumab in patients with moderate-to-severe psoriasis has been demonstrated in clinical trials and observational registries [13-15]. ESPRIT (NCT00799877) is an ongoing 10-year international prospective observational registry evaluating the long-term safety and effectiveness of originator adalimumab prescribed in routine clinical practice according to local product labeling for adult patients with chronic plaque psoriasis [13]. The objective of this analysis is to report the long-term safety, effectiveness, and PROs following treatment with adalimumab over the first 7 years of the ESPRIT registry.

\section{METHODS}

\section{Study Design and Patients}

Patient enrollment was initiated on 26 September 2008 and completed on 8 November 2012. As of 30 November 2015, 6066 patients were enrolled in the ESPRIT registry, and 6051 were analyzed. Study sites were located in the USA, Canada, Austria, Czech Republic, Denmark, France, Germany, Greece, Ireland, Netherlands, Spain, Sweden, and UK. Adalimumab was dosed as recommended in the local product label.

Adult patients ( $\geq 18$ years) with chronic plaque psoriasis prescribed adalimumab (Humira ${ }^{\circledR}$, Abbvie, Inc.) according to local product labeling and met one of the following criteria-(1) previously initiated on adalimumab therapy and having continued on adalimumab with no more than 70 consecutive days off drug or (2) newly initiated on adalimumab therapy within 4 weeks of registry entry-were eligible to be included in the ESPRIT registry. In patients who previously initiated adalimumab therapy, the initial adalimumab dose was received either in a pre-registry feeder clinical trial sponsored by AbbVie Inc, North Chicago, IL, or from an existing prescription outside of a pre-registry feeder trial. Source documentation of serious adverse events (SAEs), adverse events (AEs) of special interest, and dosing information since the initiation of therapy were provided by the physician. An independent or central ethics committee, or central or local institutional review board, approved the study. All procedures followed were in accordance with the ethical standards of the responsible committee on human experimentation (institutional and national) and with the Helsinki Declaration of 1964, as revised in 2013. Informed consent was obtained from all patients for being included in the study. 


\section{Statistical Analyses}

This analysis included two patient populations from the registry: (1) the all-treated (All-Rx) population included patients who received at least one dose of adalimumab in the registry; (2) the new-prescription (New-Rx) population, a subpopulation of All-Rx, included patients who newly initiated adalimumab within 4 weeks prior to registry enrollment. The study design and patient populations of the ESPRIT observational registry as of 30 November 2015 are illustrated in Fig. 1. Patients were evaluated at 3 and 6 months post enrollment and then every 6 months for up to 10 years. Patients were followed at intervals determined by routine clinical practice or as recommended by national guidelines. Safety data were captured throughout the entire study period. Patients who discontinued the registry drug were encouraged to remain in the registry.

Descriptive statistics are presented for baseline patient demographics and disease characteristics. Overall exposure to adalimumab (outside of and within the registry) was calculated as the time from the initial (first ever) adalimumab dose to 14 days after the last adalimumab dose in the registry, excluding the total number of days of treatment interruption in the registry. Registry exposure to adalimumab
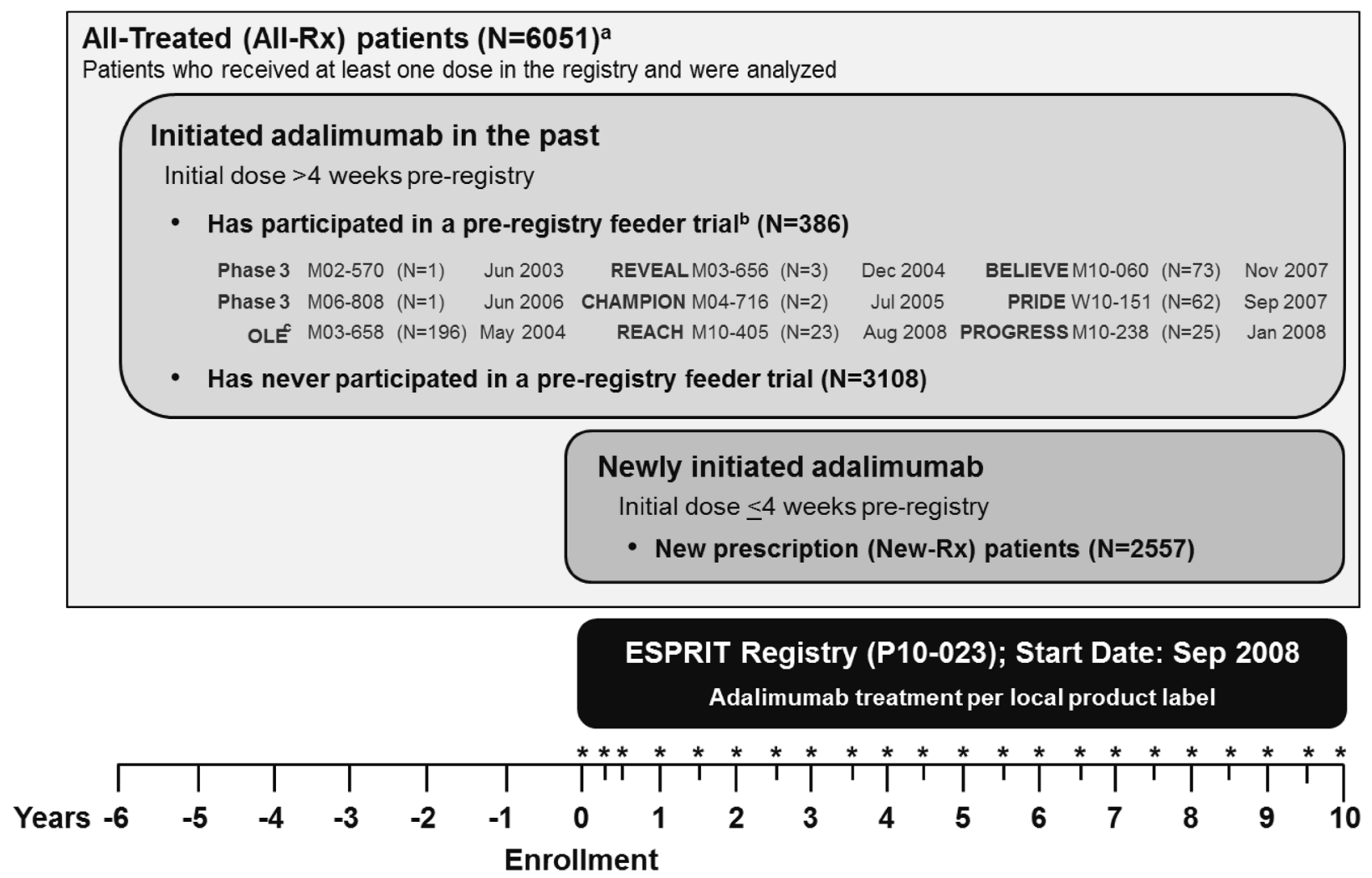

Fig. 1 Study design and patient population of the ESPRIT observational registry. ${ }^{\mathrm{a}} \mathrm{O}$ the 6066 patients enrolled, 4 had dosing information unavailable in the interim analysis of the database, and 11 patients from one site in the US were excluded from all analyses since the physician was unavailable to confirm the safety information and data in the database. ${ }^{\mathrm{b}}$ Start dates are shown for clinical trials. ${ }^{\circ}$ REVEAL and CHAMPION were among the feeder trials for the OLE; the number of patients in these trials were counted separately from OLE's 196 patients. ${ }^{*}$ Patients were evaluated 3 and 6 months post enrollment and then every 6 months for up to 10 years. Patients were followed at intervals determined by routine clinical practice or as recommended by national guidelines. Safety data are captured during the entire study period. Patients who discontinued the registry drug were encouraged to remain in the registry 
(within the registry) was calculated as the time from first adalimumab dose in the registry to 14 days after the last adalimumab dose in the registry, excluding the total number of days of treatment interruption in the registry. A treatment interruption is defined as $>70$ days without any adalimumab dose. The times to discontinuation from the registry and from the registry drug for the All-Rx and New-Rx populations were analyzed by the Kaplan-Meier method.

All-TE AEs were events occurring from the date of the initial (first ever) adalimumab dose through 70 days (5 half-lives of adalimumab) after the date of the last adalimumab dose in the registry [including AEs retrospectively collected since the initial (first ever) dose of adalimumab and from previous adalimumab studies for rollover patients], excluding AEs occurring during treatment interruptions (defined as $>70$ days without any adalimumab dose). Incidence rates for All-TEAEs are reported as events per 100 patient years of overall exposure to adalimumab (E/100PY). Incidence rates of All-TE AEs of interest by time of event occurrence in the registry are summarized for the All-Rx patient population. AEs of special interest in this analysis included cardiovascular events, serious infections, and malignancies. Cardiovascular-related AEs of special interest included myocardial infarction (MI), cerebrovascular accident (CVA), or congestive heart failure (CHF). The times to first All-TE cardiovascular event, serious infection, and malignancy were evaluated by the method of Kaplan-Meier. Standardized mortality ratio (SMR) was calculated as the ratio of observed to expected treatment-emergent deaths using the 2006 country-specific World Health Organization (WHO) mortality rates.

Treatment effectiveness was defined as patients achieving Physician's Global Assessment (PGA) of "clear" or "minimal" during registry participation (patients were not necessarily receiving adalimumab at the time of assessment). Patient-reported Dermatology Life Quality index (DLQI) and Work Productivity and Activity Impairment (WPAI) were analyzed during registry participation in US patients only. The proportion of patients achieving PGA "clear" or "minimal" and change from baseline in DLQI and WPAI scores were calculated for each registry visit in the as-observed population.

\section{RESULTS}

\section{Patient Disposition and Baseline Characteristics}

Of the 6051 patients (2557 New-Rx patients, $42.3 \%$ ) included in this 7-year interim analysis of the ESPRIT registry, a majority of patients were from sites in the US $(69.5 \%)$ and Canada (13.9\%). As of 30 November 2015, 4242 (70.1\%) All-Rx and 1652 (64.6\%) New-Rx patients were continuing in the registry. Among patients continuing in the registry, 3070 (50.7\%) All-Rx and 1061 (41.5\%) New-Rx patients have not permanently discontinued adalimumab. Of those, 2150 (35.5\%) All-Rx and 674 (26.4\%) New-Rx patients never interrupted adalimumab treatment for longer than 70 days. The most frequent reason for discontinuation from the registry was being lost to follow-up (14.4 and $19.2 \%$ for All-Rx and New-Rx populations, respectively). The reasons for discontinuation from the registry and from the registry drug in greater than $1 \%$ of All-Rx patients are listed in Table 1.

Patient demographics and disease characteristics at registry entry (baseline) were generally comparable between the All-Rx and New-Rx populations (Table 2). The majority of patients were white and younger than 65 years; 58 and $54 \%$ of All-Rx and New-Rx patients, respectively, were male. At registry entry, a greater proportion of New-Rx patients had severe or very severe disease $(36.2 \%$ vs. $19.7 \%$ for All-Rx population), as assessed by PGA, most likely because a majority of patients in the All-Rx population had received adalimumab longer than 4 weeks before registry entry. The most prevalent comorbidities observed among New-Rx patients were hypertension $(22.2 \%)$, hyperlipidemia (11.5\%), diabetes mellitus (9.7\%), and depression (9.7\%). 
Table 1 Reasons for discontinuation from the registry and from the registry drug (All-Rx and New-Rx patient population)

\begin{tabular}{|c|c|c|}
\hline Reason for discontinuation (in $>1 \%$ patients) & All-Rx $(n=6051) n(\%)$ & New-Rx $(n=2557) n(\%)$ \\
\hline From registry ${ }^{a}$, any reason & $1809(29.9)$ & $905(35.4)$ \\
\hline Lost to follow-up & $874(14.4)$ & $491(19.2)$ \\
\hline Lack of efficacy & $89(1.5)$ & $56(2.2)$ \\
\hline Withdrew consent & $355(5.9)$ & $156(6.1)$ \\
\hline Death & $70(1.2)$ & $25(1.0)$ \\
\hline Non-compliance & $86(1.4)$ & $49(1.9)$ \\
\hline Other & $333(5.5)$ & $116(4.5)$ \\
\hline From registry drug ${ }^{\mathrm{b}}$, any reason & $2981(49.3)$ & $1496(58.5)$ \\
\hline $\mathrm{AE}$ & $170(2.8)$ & $87(3.4)$ \\
\hline SAE or SAE of interest & $101(1.7)$ & $40(1.6)$ \\
\hline Lost to follow-up & $399(6.6)$ & $226(8.8)$ \\
\hline Lack of efficacy & $1101(18.2)$ & $610(23.9)$ \\
\hline Intolerance & $44(0.7)$ & $35(1.4)$ \\
\hline Withdrew consent & $117(1.9)$ & $59(2.3)$ \\
\hline Other & $445(7.4)$ & $220(8.6)$ \\
\hline Unknown reason & $668(11.0)$ & $268(10.5)$ \\
\hline
\end{tabular}

$A l l-R x$ all-treated patient population, $N e w-R x$ new prescription patient population, $A E$ adverse event, $S A E$ serious adverse event

${ }^{a}$ Reasons for registry discontinuations in $\leq 1 \%$ of patients were AE, intolerance, patient moved, SAE or SAE of interest, satisfactory improvement, pregnancy, or unknown reasons

b Reasons for registry drug discontinuations in $\leq 1 \%$ of patients were intolerance, patient death, satisfactory improvement, or required additional therapy

The median duration of overall exposure to adalimumab was 1398 (14-4798) days and 714 (14-2581) days for the All-Rx and New-Rx patient population, respectively. The median duration of registry exposure to adalimumab was 1132 (14-2581) days and 714 (14-2581) days for the All-Rx and New-Rx patient population, respectively. The number of patients according to duration of overall exposure to adalimumab and registry exposure to adalimumab is shown in Fig. 2. The time to discontinuation from the registry and from the registry drug (adalimumab) in the All-Rx and New-Rx patients is shown in Fig. 3.

\section{Safety}

This analysis included 6051 All-Rx patients representing 23,660.1 PY of overall exposure to adalimumab. An overview of incidence rates (E/ 100PY) of All-TEAEs in All-Rx patients by the duration of overall exposure to adalimumab at the time of onset of $\mathrm{AE}$ is presented in Table 3. The incidence rate of All-TE serious AEs was 4.4 E/100 PY of overall exposure to adalimumab; the most common All-TE serious AE was serious infection (1.0 E/100 PY). All-TE serious AEs with incidence of $\geq 20$ events overall were cellulitis (31 events, $0.1 \mathrm{E} / 100 \mathrm{PY}$ ), pneumonia (29 
Table 2 Patient demographics and disease characteristics at registry entry (All-Rx and New-Rx patient population)

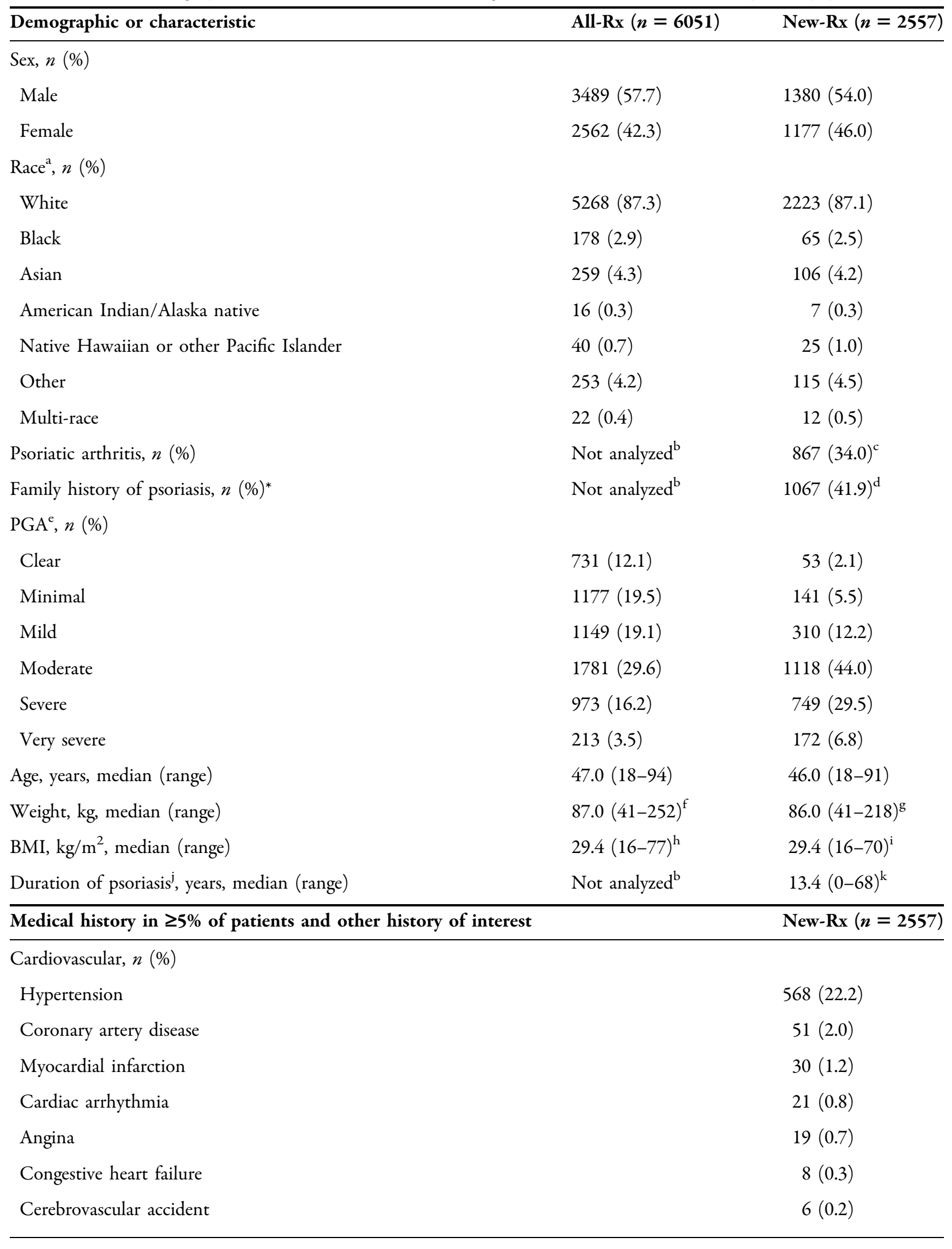


Table 2 continued

\section{Medical history in $\geq 5 \%$ of patients and other history of interest}

Gastrointestinal, $n$ (\%)

Gastroesophageal reflux disease

Inflammatory bowel disease

Metabolic

Hyperlipidemia

Diabetes mellitus

Hypothyroidism

$142(5.6)$

Obesity

Infections

Tuberculosis

Malignancy

Cancer

Neurologic and psychiatric

Depression

Anxiety disorder

Percentages calculated on non-missing values

All-Rx all-treated patient population, $N e w-R x$ new prescription patient population, $P G A$ physician's global assessment, $B M I$ body mass index

${ }^{a}$ Missing data: All-Rx: $n=15$; New-Rx: $n=4$

b Not analyzed because not all data were captured in the registry database

c Missing data: New-Rx: $n=7$

d Missing data: New-Rx: $n=9$

e Many patients had received adalimumab prior to entering the registry, demonstrated by the number of patients entering the registry with a PGA of clear or minimal (missing data: All-Rx: $n=27$; New-Rx: $n=14$ )

f $n=5927$

g $n=2497$

h $n=5909$

i $n=2492$

j Calculated at registry entry

k $n=2548$

events, 0.1 E/100 PY), and myocardial infarction (22 events, <0.1 E/100 PY). The rate of All-TE AEs leading to discontinuation of adalimumab was 1.7 E/100 PY overall, and the rates either decreased or remained stable over time. The incidence rate for All-TE AEs leading to death regardless of causality in the All-Rx population was $0.1 \mathrm{E} / 100 \mathrm{PY}$; the most common event leading to death was myocardial infarction (five events, <0.1 E/100 PY).

The incidence rates of All-TE inflammatory bowel disease [Crohn's disease, four events (two patients with documented history of Crohn's disease); ulcerative colitis, two events] and depression (34 events) were $<0.1 \mathrm{E} / 100 \mathrm{PY}$ and $0.1 \mathrm{E} / 100 \mathrm{PY}$, respectively. Incidence rates for 


\section{Number of patients based on their overall exposure to ADA (outside of and within the registry)}

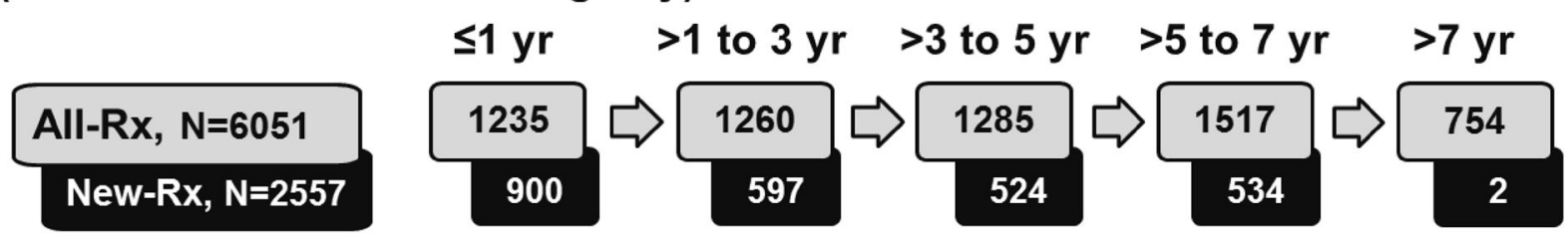

\section{Number of patients based on their registry exposure to ADA (within the registry)}

\section{All-Rx, $\mathrm{N}=6051$ \\ New-Rx, N=2557}

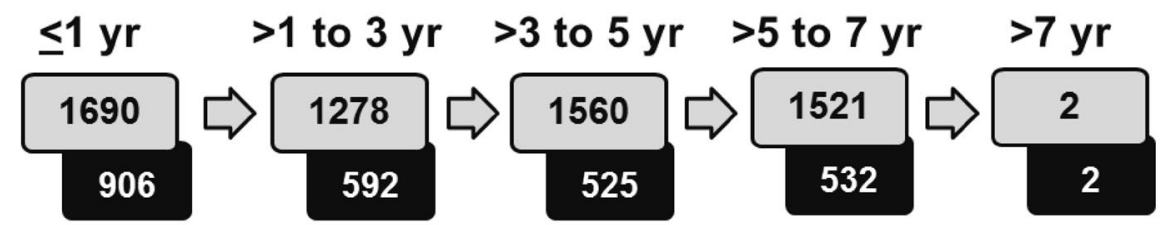

Fig. 2 Number of patients based on duration of adalimumab exposure (All-Rx and new-Rx patient population). Overall exposure to adalimumab (outside of and within the registry) was calculated as the time from the initial (first ever) adalimumab dose to 14 days after the last adalimumab dose in the registry, excluding the total number of days of treatment interruption in the registry. Registry exposure to adalimumab (within the registry) was

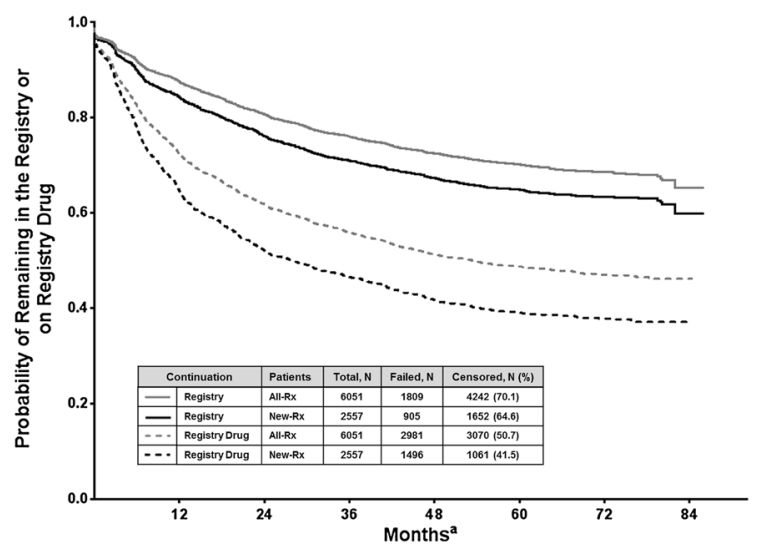

Fig. 3 Kaplan-Meier plots of time to discontinuation from the registry and from the registry drug (adalimumab) (All-Rx and New-Rx patient populations). ${ }^{a}$ Time of observation (first day to last day of registry participation) was used for time to registry discontinuation; registry exposure to adalimumab was used for time to registry drug discontinuation. All-Rx all-treated patient population, $N e w-R x$ new prescription patient population calculated as the time from the first adalimumab dose in the registry to 14 days after the last adalimumab dose in the registry, excluding the total number of days of treatment interruption (defined as $>70$ days without any adalimumab dose) in the registry. $A D A$ adalimumab; All-Rx all-treated patient population, New-Rx new prescription patient population

All-TE hyperlipidemia (12 events), diabetes mellitus (21 events), hypothyroidism (7 events), and obesity (4 events) were $<0.1 \mathrm{E} / 100 \mathrm{PY}$ each. The incidence rates of arthritis (16 events), psoriatic arthritis (44 events), rheumatoid arthritis (4 events), and myalgia (23 events) were $<0.1, \quad 0.1,0.2,<0.1$, and $<0.1 \mathrm{E} / 100 \mathrm{PY}$, respectively.

An overview of incidence rates (E/100 PY) for All-TE cardiovascular events, serious infections, and malignancies in All-Rx patients by the duration of overall exposure to adalimumab at the time of onset of the $\mathrm{AE}$ is shown in Fig. 4. All-TE cardiovascular events, serious infections, and malignancies were the primary causes of 8 , 1 , and 12 deaths $(<0.1 \mathrm{E} / 100 \mathrm{PY}$, each), respectively.

The time to first All-TE occurrence of myocardial infarction, cerebrovascular accident, congestive heart failure, serious infection, malignancy, lymphoma, or non-melanoma skin cancer in the All-Rx patient population is 


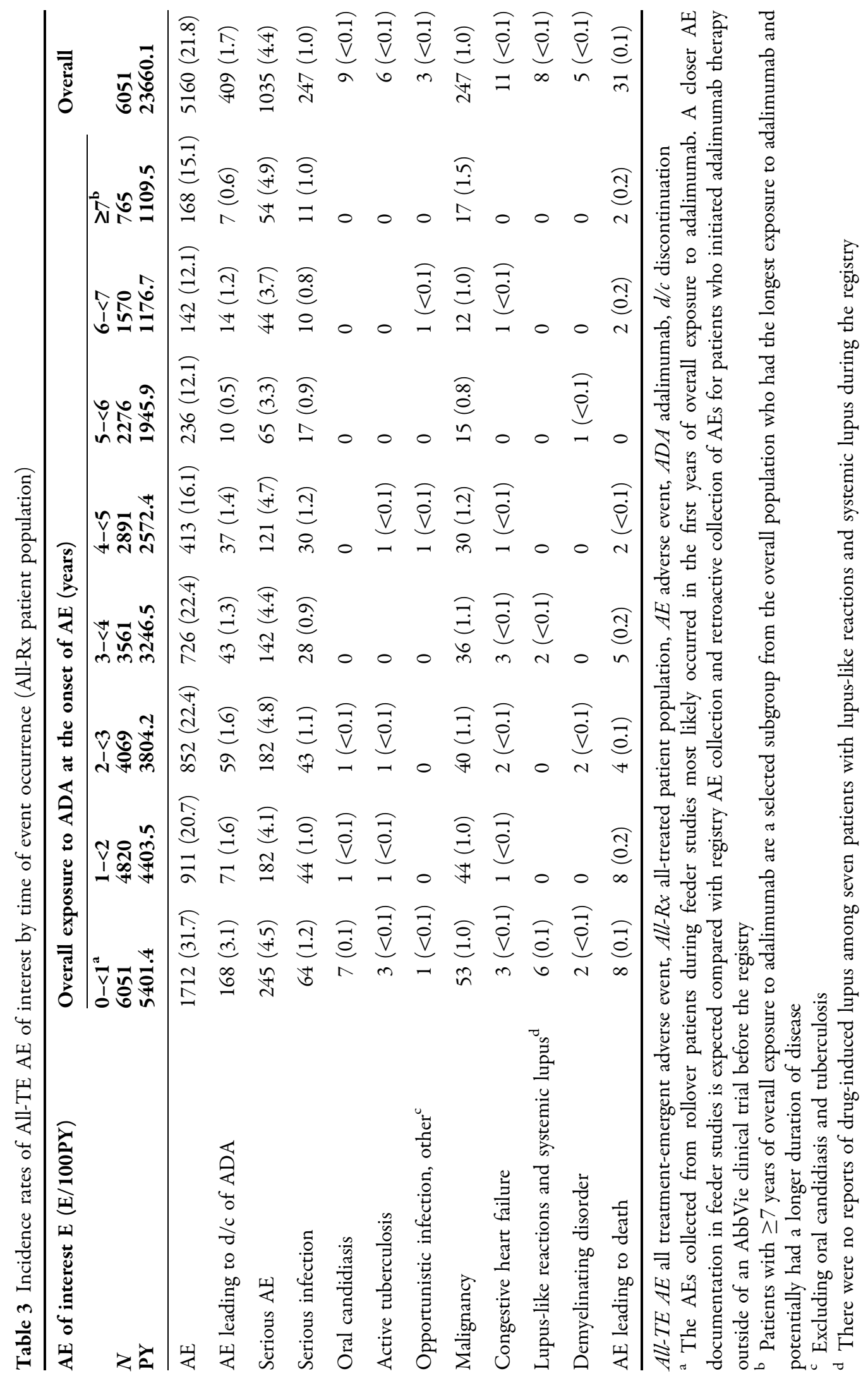




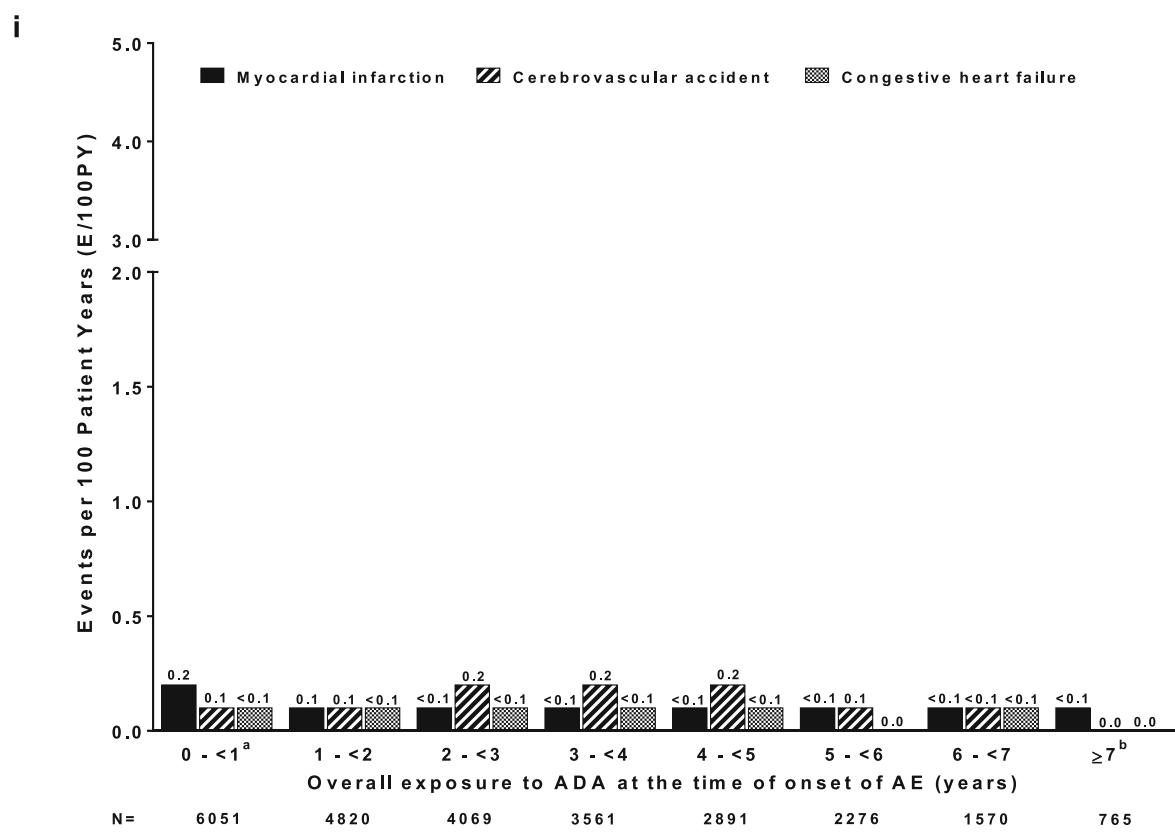

ii

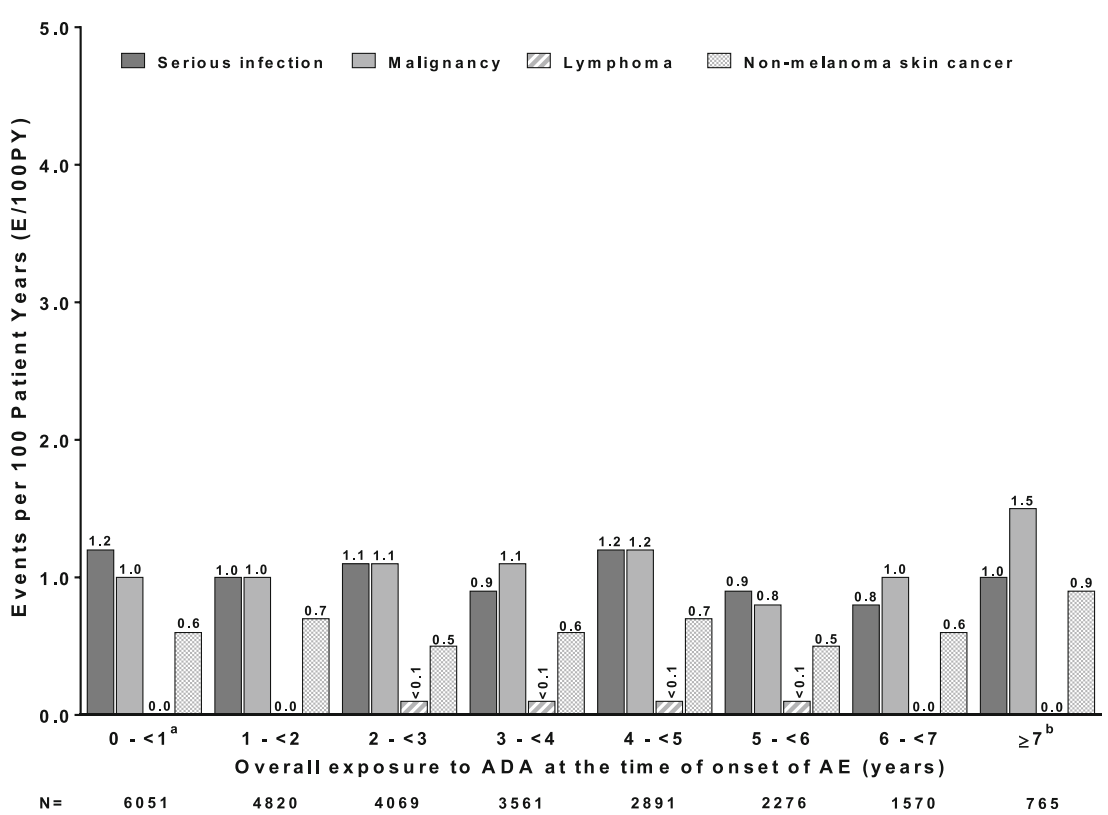

Fig. 4 Incidence rates of All-TE cardiovascular events (1) and serious infections and malignancies (2) by time of event occurrence (All-Rx patient population). ${ }^{a}$ The AEs collected from rollover patients during feeder studies most likely occurred in the first years of overall exposure to adalimumab. A closer AE documentation in feeder studies is expected compared with registry $\mathrm{AE}$ collection and retroactive collection of AEs for patients who initiated adalimumab therapy outside of an AbbVie clinical trial before the registry. ${ }^{b}$ Patients with $\geq 7$ years of overall exposure to adalimumab are a selected subgroup from the overall population who had the longest exposure to adalimumab and potentially had a longer duration of disease. All-TE AE all treatment-emergent adverse event, All-Rx all-treated patient population, $M I$ myocardial infarction, $C V A$ cerebrovascular accident, $C H F$ congestive heart failure, $S I$ serious infection, $N M S C$ non-melanoma skin cancer, $A D A$ adalimumab, $A E$ adverse event 


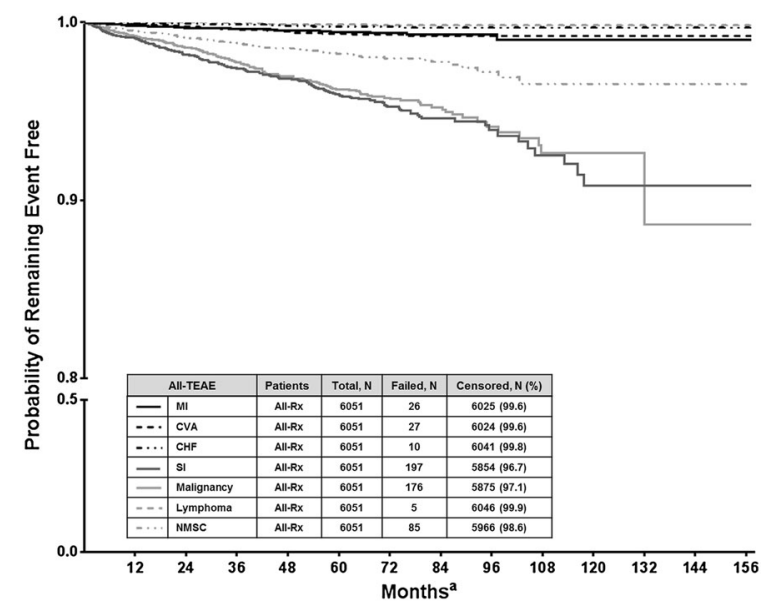

Fig. 5 Time to first all-TE AE of interest (All-Rx patient population). ${ }^{a}$ Overall exposure to adalimumab (outside of and within the registry). All-TE $A E$ all treatment-emergent adverse event, All-Rx all-treated patient population, $M I$ myocardial infarction, $C V A$ cerebrovascular accident, $C H F$ congestive heart failure, $S I$ serious infection, NMSC non-melanoma skin cancer

shown in Fig. 5. Through 30 November 2015, $99.6 \%, 99.6 \%, 99.8 \%, 96.7 \%$, and $97.1 \%$ of patients remained free of All-TE myocardial infarction, cerebrovascular accident, congestive heart failure, serious infections, and malignancies, respectively. The standardized mortality ratio was 0.27 (95\% CI 0.18-0.38) for All-Rx and 0.28 (95\% CI 0.14-0.50) for New-Rx, indicating that the observed number of deaths in the ESPRIT registry was below expected for the age-, sex- and country-matched population (Fig. 6).

\section{Effectiveness and Patient-Reported Outcomes}

During the first 7 years of registry participation, over $50 \%$ of All-Rx and New-Rx patients achieved PGA "clear" or "minimal" (Fig. 7, patient numbers were small at 84 months). However, patients were not necessarily receiving adalimumab at the time of assessment. The proportions of All-Rx and New-Rx patients achieving PGA "clear" or "minimal" were similar at each time point and generally increased through 7 years.

Among US patients who reported DLQI data, mean baseline DLQI scores were 7.6 (95\% CI

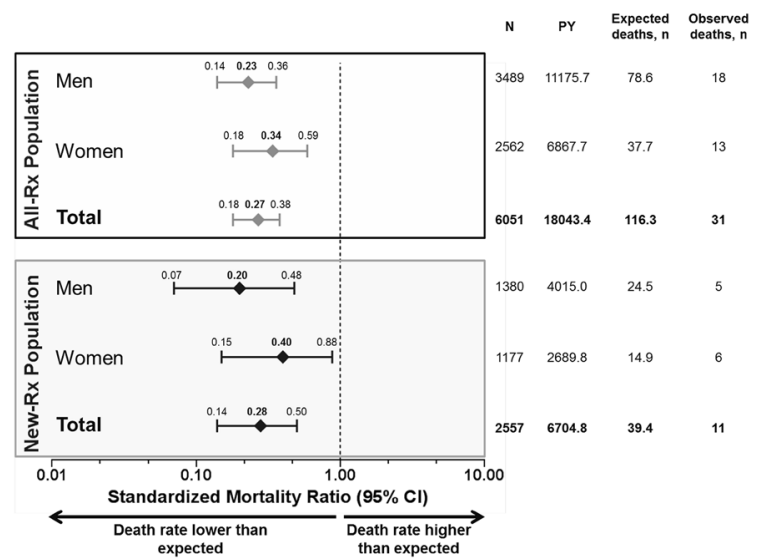

Fig. 6 Standardized mortality ratios $(\mathrm{SMR})^{\mathrm{a}}$, overall and by gender (All-Rx and new-Rx patient population). ${ }^{a} S M R$ was calculated as the ratio of observed to expected treatment-emergent deaths using the 2006 country-specific World Health Organization (WHO) mortality rates. All-Rx all-treated patient population, New-Rx new prescription patient population, $P Y$ patient years, $C I$ confidence intervals

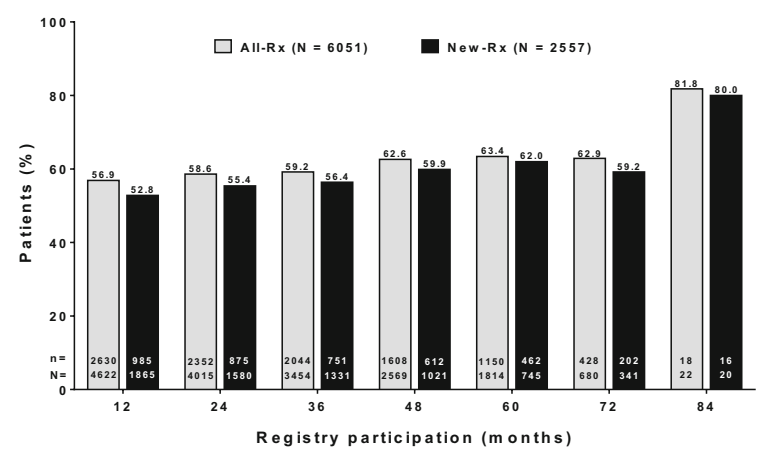

Fig. 7 Proportion of patients (as observed) achieving PGA "clear" or "minimal" (All-Rx and new-Rx patient population). Patients were not necessarily receiving adalimumab at the time of PGA assessment. Only a few selected patients left at 84 months. $P G A$ physician's global assessment, All-Rx all-treated patient population, New-Rx new prescription patient population

7.4-7.8) and 10.9 (95\% CI 10.5-11.2) in All-Rx $(n=4084)$ and New-Rx $(n=1887)$ patient populations, respectively. The mean change from baseline in DLQI scores was at least -3.1 in All-Rx patients and -5.5 in New-Rx patients (Fig. 8). The improvements in DLQI scores from baseline were maintained through the first 7 years of the registry. Among US patients, 


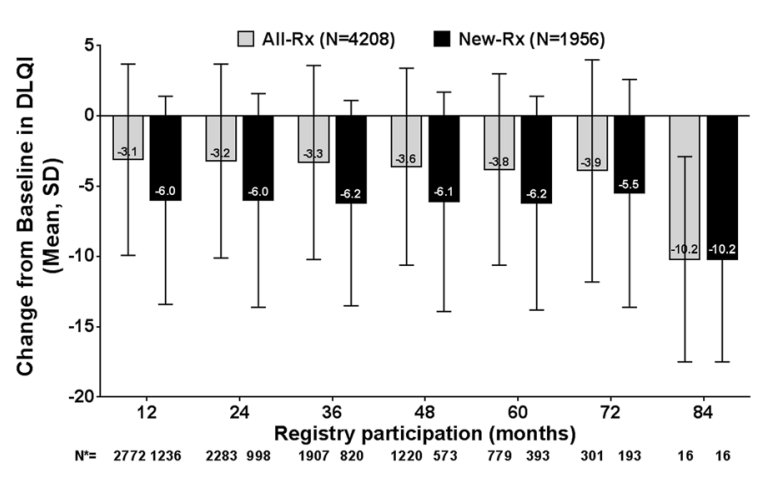

Fig. 8 Change from baseline (as observed) in DLQI scores $^{\mathrm{a}}$ (All-Rx and new-Rx patient population, US patients only). a Decrease in DLQI scores denotes improvement. $N^{*}$ number of patients in as-observed population for respective visit; only a few selected patients left at 84 months. PGA physician's global assessment, All-Rx all-treated patient population, New-Rx new prescription patient population

improvements in total work productivity impairment (TWPI), total activity impairment (TAI), presenteeism, and absenteeism were observed during the first 7 years of the ESPRIT registry (Fig. 9). The improvements in WPAI scores from baseline were maintained through the first 7 years of the registry.

\section{DISCUSSION}

During the first 7 years of the ESPRIT registry, no new or unexpected safety signals were observed with adalimumab treatment, and the safety was consistent with the known safety profile of adalimumab. The safety results from the ESPRIT registry were similar to those from the integrated safety analysis of the adalimumab clinical trials in psoriasis and across indications [14-16]. At registry entry, baseline characteristics were similar between All-Rx and New-Rx populations with the exception of greater disease severity in the New-Rx population, which could be attributed to a majority of patients in the All-Rx population receiving adalimumab for longer than 4 weeks at baseline and therefore having better disease control at registry entry. The most frequently reported psoriasis-related comorbidities at enrollment were hypertension, hyperlipidemia,
Fig. 9 Change from baseline (as observed) in WPAI scores $^{\mathrm{a}}$ (All-Rx and New-Rx patient population, US patients only). ${ }^{a}$ Decrease in WPAI subscores denotes improvement. $N^{*}=$ number of patients in as-observed population for respective visit; only a few selected patients left at 84 months. WPAI work productivity and activity impairment, TWPI total work productivity impairment, TAI total activity impairment, presenteeism impairment while working, All-Rx all-treated patient population, $N e w-R x$ new prescription patient population

diabetes mellitus, and depression. Although the All-Rx patient population had longer overall exposure to adalimumab compared with New-Rx population, similar proportions of patients continued in the registry and received adalimumab.

The incidence rates of All-TE AEs by the duration of overall exposure to adalimumab at the time of onset of AE generally remained stable over 7 years in the ESPRIT registry. Intolerance to initial adalimumab therapy and subsequent discontinuations most likely occurred during the first year of adalimumab. In addition, patients with $\geq 7$ years of overall exposure to adalimumab are a selected subgroup from the overall population who had the longest exposure to adalimumab and potentially had a longer duration of disease.

Patients with moderate-to-severe psoriasis may have an elevated background risk of cardiovascular events, serious infections, and malignancies compared with general population $[3,6,8,9,17,18]$. Although some studies have reported increased risk of serious infections or malignancies, particularly lymphoma in psoriasis patients treated with TNF- $\alpha$ antagonists, significantly increased risks have not been identified in other studies of TNF- $\alpha$ antagonists [19-22]. In addition, patients with psoriasis receiving TNF- $\alpha$ antagonists have been found to be at reduced risk for cardiovascular events [23-27].

Incidence rates of treatment-emergent cardiovascular events, serious infections, and malignancies in the ESPRIT registry were consistent with rates observed in the adalimumab clinical trials, and there was no cumulative effect for up to $>7$ years of total adalimumab 
i

All-Rx, US Patients Only $(\mathrm{N}=4208)$

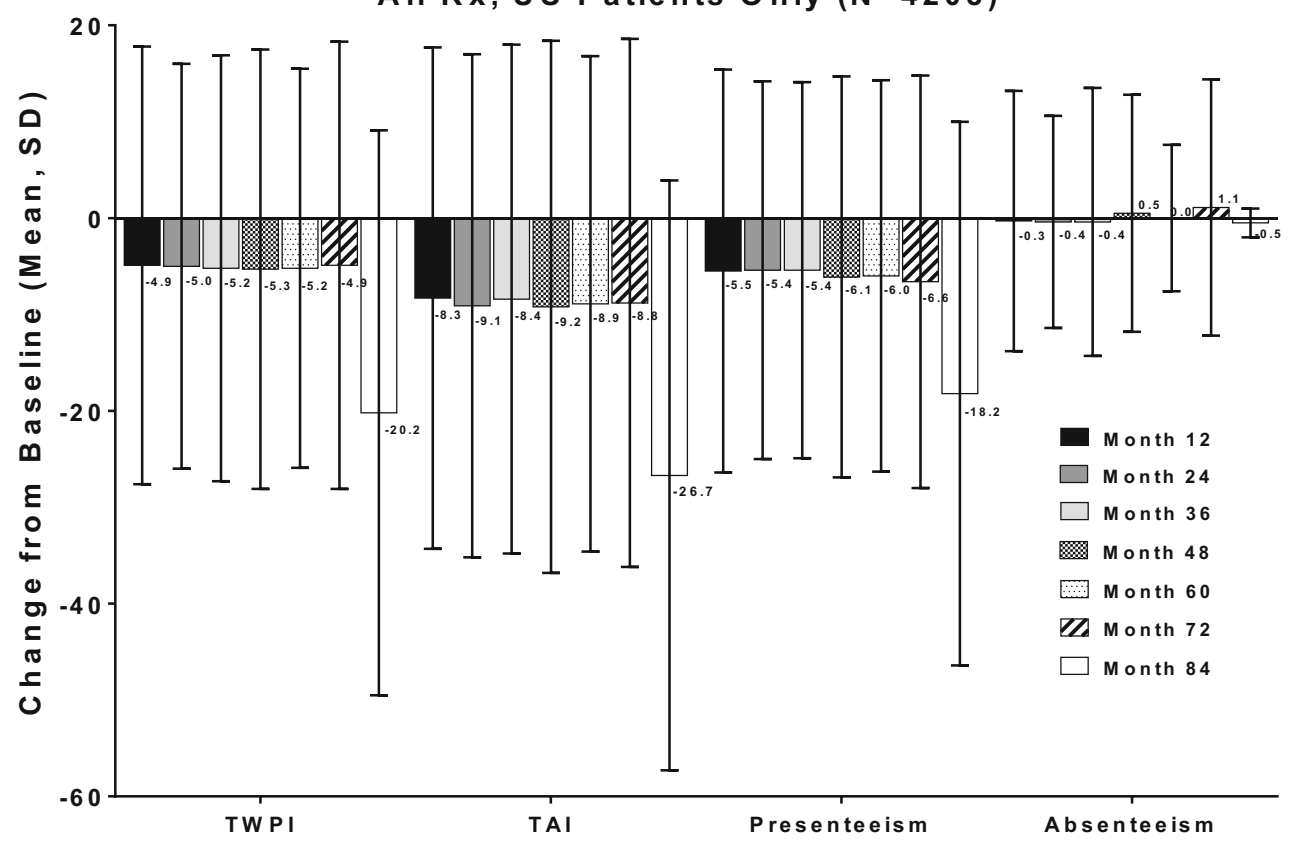

Work Productivity and Activity Impairment (Measured in \%)

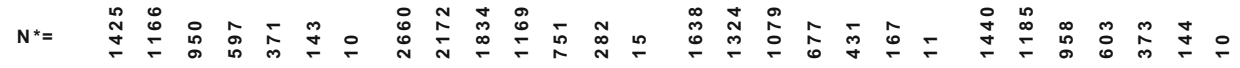

i i

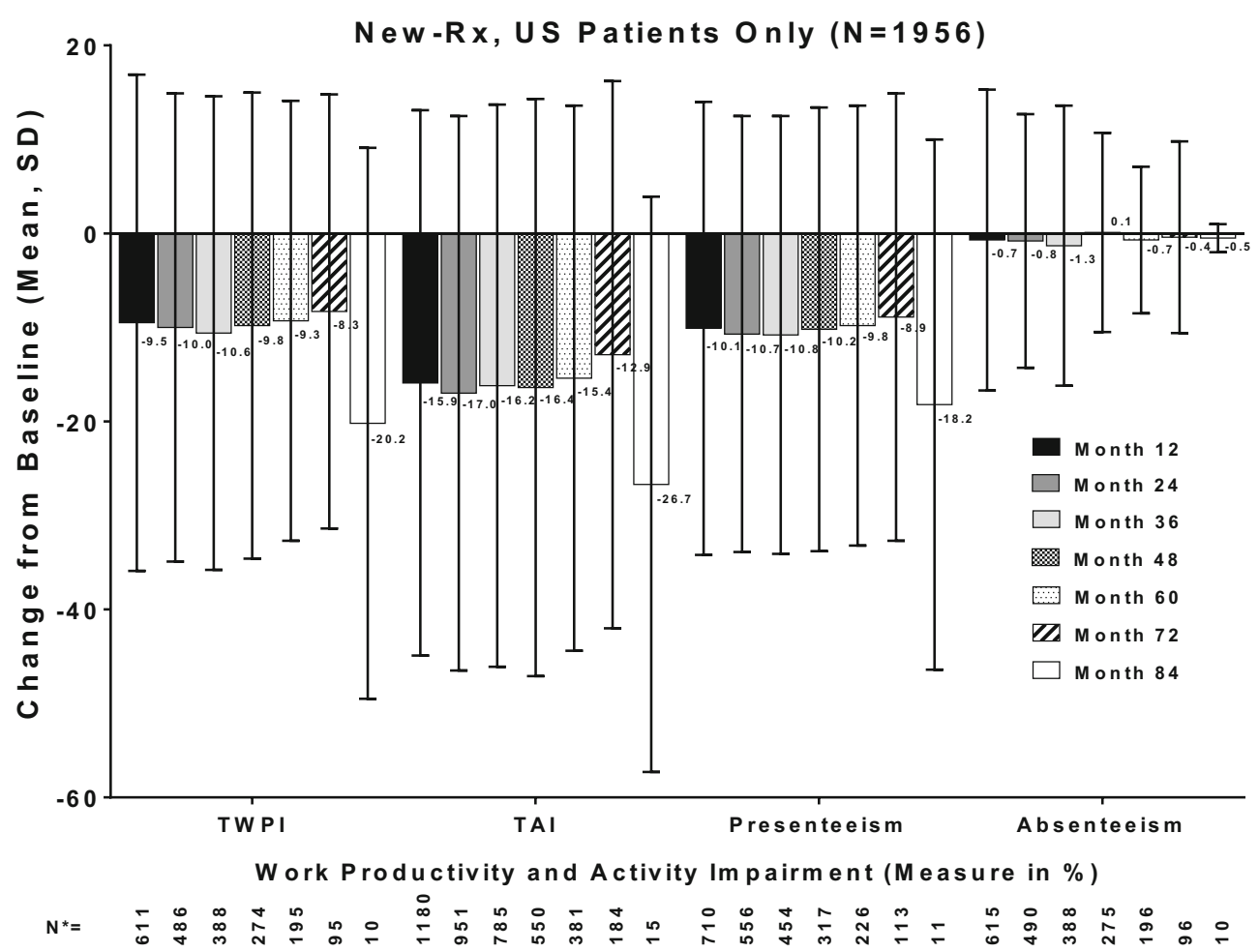


exposure. As of 30 November 2015, most of the patients ( $>96 \%)$ remained free of All-TE cardiovascular events, serious infections, or malignancies. The observed incidence rates in the ESPRIT registry were within ranges of published rates of cardiovascular events, serious infections, and malignancies in psoriasis patients not receiving biologic treatment (0.6-1.5 E/100 PY, 0.3-2.1 E/100 PY, and 0.5-2.0 $\mathrm{E} / 100 \mathrm{PY}$, respectively $[10,21,28-32])$ and consistent with the expected rate in the psoriasis population treated with TNF- $\alpha$ antagonists $[21,22,33]$. The number of observed treatment-emergent deaths in the ESPRIT registry was below the expected rate for the age-, sex-, and country-matched general population. This lower risk could be partly due to patient selection bias as patients at a higher risk of mortality probably tended to be not enrolled into the registry.

As-observed effectiveness of adalimumab either increased or remained stable through 7 years of the ESPRIT registry, even though some patients may have entered the registry with PGA score of "clear" or "minimal," having received adalimumab treatment for longer than 4 weeks. Since the registry was designed to understand the long-term effectiveness of adalimumab outside of a controlled trial setting and even when adalimumab was stopped, the results may be confounded by patients not necessarily receiving adalimumab at the time of assessment and discontinuations due to lack of efficacy. During the first 7 years of the ESPRIT registry, improvements in DLQI and WPAI scores were maintained in both patient populations.

Some of the limitations of this analysis are the lack of a control group for comparison and that observational data are subject to outcome-reporting bias. At registry entry, some patients did not have previous exposure to adalimumab. In addition, at the time of assessment of effectiveness and PROs, patients were not necessarily receiving adalimumab. The overall exposure-adjusted incidence rates of All-TE AEs may be underestimated in patients who were receiving adalimumab long-term prior to registry entry.

\section{CONCLUSIONS}

In summary, no new safety signals were observed with adalimumab treatment in the first 7 years of the ESPRIT registry. Safety data were consistent with the known safety profile of adalimumab from prior clinical trials and postmarketing surveillance. The number of treatment-emergent deaths in the registry was below the expected rate for the comparable general population. The majority of patients remained free of treatment-emergent cardiovascular events, serious infection, or malignancy for up to 7 years of overall registry exposure to adalimumab. As-observed effectiveness of adalimumab and improvement from baseline in PROs remained stable through 7 years of the registry.

\section{ACKNOWLEDGEMENTS}

AbbVie funded the ESPRIT registry (NCT00799877), contributed to its design, and participated in the data collection, analysis and interpretation of the data and in the writing, review, and approval of the publication. Article processing charges were funded by AbbVie. All authors had full access to all of the data in this study and take complete responsibility for the integrity of the data and accuracy of the data analysis. All named authors meet the International Committee of Medical Journal Editors (ICMJE) criteria for authorship for this manuscript, take responsibility for the integrity of the work as a whole, and have given final approval to the version to be published. Medical writing support was provided by Deepa Venkitaramani, $\mathrm{PhD}$, of AbbVie. AbbVie and the authors thank all study investigators for their contributions and the patients who participated in this study.

Disclosures. A Menter has grants and honoraria from AbbVie, Amgen, Janssen Biotech, Inc., and LEO Pharma for service on an advisory board, as consultant, investigator, and speaker; received grants and honoraria from Allergan for service on an advisory board and as a consultant and from Eli Lilly for service on an 
advisory board, as a consultant and investigator; received grants and honoraria from Boehringer Ingelheim for service on an advisory board and as an investigator; received grants and honoraria from Novartis and Pfizer for service as a consultant and investigator; received grants from Celgene, Dermira, Merck, Neothetics, Regeneron, and Syntrix for service as an investigator and received honoraria from Galderma for service as a consultant. D Thaçi has received honoraria from AbbVie, Amgen, Biogen-Idec, Celgene, GSK, Dignity, Janssen, Leo, Maruho, Mitsubishi, Lilly, Novartis, Pfizer, Regeneron/ Sanofi, and UCB for participation on ad boards, as a speaker, and for consultancy and received research grants from AbbVie, Biogen-Idec, Leo, and Pfizer. JJ Wu has received research funding from AbbVie, Amgen, Eli Lilly, Janssen, Novartis, and Regeneron. W Abramovits has received honoraria or fees for serving on advisory boards, as a speaker, as a consultant, grants as an investigator from AbbVie, Allergan, Amgen, Anacor Pharm, Aqua Pharma, Celgene, Centocor, Conversant, Eli Lilly, Exeltis, Galderma, Genentech, Glenmark, GSK, Innocutis, Janssen Biotech, Leo Pharma, MediMetricks, Merck Serono, Novartis, Novan, Otsuka, PharmaDerm, Perrigo, Pfizer, Promius, Prothena, PuraCap, Quinnova, Ranbaxy, Regeneron, Sanofi, Taro, Teva, Tioga, Tolmar, Valeant, and Xenoport. $F$ Kerdel has received honoraria from AbbVie, Amgen, Celgene, Janssen, Leo, Pfizer, Eli Lilly, Novartis, Stiefel, and Valeant for participation as a speaker and received grants from AbbVie, Amgen, AstraZeneca, Celgene, Janssen, Eli Lilly, Novartis, and Pfizer, for participation as an investigator. D Arikan is a full-time salaried employee of AbbVie and may own stock/options. D Guo is a full-time salaried employee of AbbVie and may own stock/options. A Ganguli is a full-time salaried employee of AbbVie and may own stock/options. $M$ Bereswill is a full-time salaried employee of AbbVie and may own stock/options. A Camez is a full-time salaried employee of AbbVie and may own stock/ options. WC Valdecantos is a full-time salaried employee of AbbVie and may own stock/ options.
Compliance with Ethics Guidelines. An independent or central ethics committee, or central or local institutional review board, approved the study. All procedures followed were in accordance with the ethical standards of the responsible committee on human experimentation (institutional and national) and with the Helsinki Declaration of 1964, as revised in 2013. Informed consent was obtained from all patients for being included in the study

Data Availability. Qualified researchers may request access to the study datasets from AbbVie via the process defined on AbbVie.com under Clinical Trial Data and Information Sharing.

Open Access. This article is distributed under the terms of the Creative Commons Attribution-NonCommercial 4.0 International License (http://creativecommons.org/licenses/ by-nc/4.0/), which permits any noncommercial use, distribution, and reproduction in any medium, provided you give appropriate credit to the original author(s) and the source, provide a link to the Creative Commons license, and indicate if changes were made.

\section{REFERENCES}

1. Boehncke W-H, Schön MP. Psoriasis. Lancet. 2015;386(9997):983-94.

2. Menter A, Korman NJ, Elmets CA, Feldman SR, Gelfand JM, Gordon KB, et al. Guidelines of care for the management of psoriasis and psoriatic arthritis: section 6. Guidelines of care for the treatment of psoriasis and psoriatic arthritis: case-based presentations and evidence-based conclusions. J Am Acad Dermatol. 2011;65(1):137-74.

3. Ryan C, Kirby B. Psoriasis is a systemic disease with multiple cardiovascular and metabolic comorbidities. Dermatol Clin. 2015;33(1):41-55.

4. Yeung H, Takeshita J, Mehta NN, Kimmel SE, Ogdie A, Margolis DJ, et al. Psoriasis severity and the prevalence of major medical comorbidity: a population-based study. JAMA Dermatol. 2013;149(10):1173-9. 
5. Boehncke WH, Menter A. Burden of disease: psoriasis and psoriatic arthritis. Am J Clin Dermatol. 2013;14(5):377-88.

6. Horreau C, Pouplard C, Brenaut E, Barnetche T, Misery L, Cribier B, et al. Cardiovascular morbidity and mortality in psoriasis and psoriatic arthritis: a systematic literature review. J Eur Acad Dermatol Venereol. 2013;27(3):12-29.

7. Greb JE, Goldminz AM, Elder JT, Lebwohl MG, Gladman DD, Wu JJ, et al. Psoriasis. Nat Rev Dis Prim. 2016;2:16082.

8. Wakkee M, de Vries E, van den Haak P, Nijsten T. Increased risk of infectious disease requiring hospitalization among patients with psoriasis: a population-based cohort. J Am Acad Dermatol. 2011;65(6):1135-44.

9. Pouplard C, Brenaut E, Horreau C, Barnetche T, Misery L, Richard MA, et al. Risk of cancer in psoriasis: a systematic review and meta-analysis of epidemiological studies. J Eur Acad Dermatol Venereol. 2013;27(Suppl 3):36-46.

10. Kimball AB, Schenfeld J, Accortt NA, Anthony MS, Rothman KJ, Pariser D. Incidence rates of malignancies and hospitalized infectious events in patients with psoriasis with or without treatment and a general population in the USA 2005-2009. Br J Dermatol. 2014;170(2):366-73.

11. Gottlieb A, Korman NJ, Gordon KB, Feldman SR, Lebwohl M, Koo JY, et al. Guidelines of care for the management of psoriasis and psoriatic arthritis: Section 2. Psoriatic arthritis: overview and guidelines of care for treatment with an emphasis on the biologics. J Am Acad Dermatol. 2008;58(5):851-64.

12. Humira. [package insert]. AbbVie Inc. North Chicago, IL. 2016

13. Menter A, Thaci D, Papp KA, Wu JJ, Bereswill M, Teixeira $\mathrm{HD}$, et al. Five-year analysis from the ESPRIT 10-year postmarketing surveillance registry of adalimumab treatment for moderate to severe psoriasis. J Am Acad Dermatol. 2015;73(3):410-419 e6.

14. Leonardi C, Papp K, Strober B, Reich K, Asahina A, $\mathrm{Gu} \mathrm{Y}$, et al. The long-term safety of adalimumab treatment in moderate to severe psoriasis: a comprehensive analysis of all adalimumab exposure in all clinical trials. Am J Clin Dermatol. 2011;12(5):321-37.

15. Gordon K, Papp K, Poulin Y, Gu Y, Rozzo S, Sasso $\mathrm{EH}$. Long-term efficacy and safety of adalimumab in patients with moderate to severe psoriasis treated continuously over 3 years: results from an open-label extension study for patients from REVEAL. J Am Acad Dermatol. 2012;66(2):241-51.

16. Burmester GR, Panaccione R, Gordon KB, McIlraith MJ, Lacerda AP. Adalimumab: long-term safety in 23,458 patients from global clinical trials in rheumatoid arthritis, juvenile idiopathic arthritis, ankylosing spondylitis, psoriatic arthritis, psoriasis and Crohn's disease. Ann Rheum Dis. 2013;72(4):517-24.

17. Samarasekera EJ, Neilson JM, Warren RB, Parnham $\mathrm{J}$, Smith $\mathrm{CH}$. Incidence of cardiovascular disease in individuals with psoriasis: a systematic review and meta-analysis. J Invest Dermatol. 2013;133(10):2340-6.

18. Gelfand JM, Neimann AL, Shin DB, Wang X, Margolis DJ, Troxel AB. Risk of myocardial infarction in patients with psoriasis. JAMA. 2006;296(14):1735-41.

19. Dommasch ED, Abuabara K, Shin DB, Nguyen J, Troxel AB, Gelfand JM. The risk of infection and malignancy with tumor necrosis factor antagonists in adults with psoriatic disease: a systematic review and meta-analysis of randomized controlled trials. J Am Acad Dermatol. 2011;64(6):1035-50.

20. Mariette X, Matucci-Cerinic M, Pavelka K, Taylor P, van Vollenhoven R, Heatley R, et al. Malignancies associated with tumour necrosis factor inhibitors in registries and prospective observational studies: a systematic review and meta-analysis. Ann Rheum Dis. 2011;70(11):1895-904.

21. Reich K, Mrowietz U, Radtke MA, Thaci D, Rustenbach SJ, Spehr C, et al. Drug safety of systemic treatments for psoriasis: results from the German Psoriasis Registry PsoBest. Arch Dermatol Res. 2015;307(10):875-83.

22. Garcia-Doval I, Cohen AD, Cazzaniga S, Feldhamer I, Addis A, Carretero G et al. Risk of serious infections, cutaneous bacterial infections, and granulomatous infections in patients with psoriasis treated with anti-tumor necrosis factor agents versus classic therapies: prospective meta-analysis of Psonet registries. J Am Acad Dermatol. 2017;76(2):299-308e16.

23. Gulliver W. Long-term prognosis in patients with psoriasis. Br J Dermatol. 2008;159(Suppl 2):2-9.

24. Famenini S, Sako EY, Wu JJ. Effect of treating psoriasis on cardiovascular co-morbidities: focus on TNF inhibitors. Am J Clin Dermatol. 2014;15(1):45-50.

25. Ahlehoff O, Skov L, Gislason G, Gniadecki R, Iversen L, Bryld LE, et al. Cardiovascular outcomes and systemic anti-inflammatory drugs in patients 
with severe psoriasis: 5-year follow-up of a Danish nationwide cohort. J Eur Acad Dermatol Venereol. 2015;29(6):1128-34.

26. Wu JJ, Poon KY, Channual JC, Shen AY. Association between tumor necrosis factor inhibitor therapy and myocardial infarction risk in patients with psoriasis. Arch Dermatol. 2012;148(11):1244-50.

27. Wu JJ, Guerin A, Sundaram M, Dea K, Cloutier M, Mulani P. Cardiovascular event risk assessment in psoriasis patients treated with tumor necrosis factor-alpha inhibitors versus methotrexate. J Am Acad Dermatol. 2017;76(1):81-90.

28. Ahlehoff $\mathrm{O}$, Gislason $\mathrm{GH}$, Charlot $\mathrm{M}$, Jorgensen $\mathrm{CH}$, Lindhardsen J, Olesen JB, et al. Psoriasis is associated with clinically significant cardiovascular risk: a Danish nationwide cohort study. J Intern Med. 2011;270(2):147-57.

29. Gottlieb AB, Kalb RE, Langley RG, Krueger GG, de Jong EM, Guenther L, et al. Safety observations in 12095 patients with psoriasis enrolled in an international registry (PSOLAR): experience with infliximab and other systemic and biologic therapies. J Drugs Dermatol. 2014;13(12):1441-8.
30. Kalb RE, Fiorentino DF, Lebwohl MG, Toole J, Poulin Y, Cohen AD, et al. Risk of serious infection with biologic and systemic treatment of psoriasis: results from the psoriasis longitudinal assessment and registry (PSOLAR). JAMA Dermatol. 2015;151(9):961-9.

31. Brauchli YB, Jick SS, Miret M, Meier CR. Psoriasis and risk of incident cancer: an inception cohort study with a nested case-control analysis. J Invest Dermatol. 2009;129(11):2604-12.

32. Paul CF, Ho VC, McGeown C, Christophers E, Schmidtmann B, Guillaume JC, et al. Risk of malignancies in psoriasis patients treated with cyclosporine: a 5 years cohort study. J Invest Dermatol. 2003;120(2):211-6.

33. Kimball AB, Rothman KJ, Kricorian G, Pariser D, Yamauchi PS, Menter A, et al. OBSERVE-5: observational postmarketing safety surveillance registry of etanercept for the treatment of psoriasis final 5-year results. J Am Acad Dermatol. 2015;72(1):115-22. 